\title{
Evolving Concepts in the Diagnosis and Management of May-Thurner Syndrome
}

\author{
Murthy R. Chamarthy ${ }^{1}$ Patrick Sutphin ${ }^{1}$ Matthew Anderson ${ }^{1} \quad$ Mark Reddick $^{1}$ Sanjeeva P. Kalva ${ }^{1}$ \\ ${ }^{1}$ Department of Radiology, University of Texas Southwestern Medical \\ Center, Dallas, Texas, United States \\ J Clin Interv Radiol ISVIR 2017;1:23-30. \\ Address for correspondence Murthy R. Chamarthy, MD, Department \\ of Radiology, University of Texas Southwestern Medical Center, 5323 \\ Harry Hines Boulevard, Dallas, TX 75390-8834, United States \\ (e-mail: Murthy.Chamarthy@UTSouthwestern.edu).
}

\begin{abstract}
Keywords

- May-Thurner Syndrome

- iliac vein compression

- stent

- IVUS

- thrombus

- thrombolysis

May-Thurner syndrome is a common cause of left lower extremity venous thrombosis resulting from repetitive and dynamic compression of the left common iliac vein by the right common iliac artery, commonly seen in young to middle-aged women. The diagnosis requires high clinical suspicion. Computed tomography venography and magnetic resonance venography depict the extent of venous thrombosis and iliac venous compression in patients with underlying thrombotic and nonthrombotic pathologies, respectively. Contrast venography allows detection of acute and chronic thrombus, associated venous obstruction, and collaterals but is of limited value to evaluate the early venous wall abnormalities. Endovascular ultrasound is highly useful to detect early mural changes and accurately quantify venous narrowing. Endovascular intervention with balloon dilatation and stenting with or without pharmacomechanical thrombectomy followed by long-term anticoagulation is the preferred treatment with excellent technical and clinical outcomes. This review focuses on the current understanding of the pathology, clinical presentation, and endovascular management of May-Thurner syndrome.
\end{abstract}

\section{Introduction}

May-Thurner syndrome (MTS) is caused by compression of the left common iliac vein by the right common iliac artery, resulting in symptoms of venous obstruction, insufficiency, and thrombosis. It is also known as iliac vein compression syndrome or Cockett's syndrome. ${ }^{1}$ McMurrich initially reported association of common iliac vein compression with increased incidence of left lower extremity deep vein thrombus, thought to be congenital. ${ }^{2}$ Ehrich postulated acquired degenerative obstruction at the origin of the left common iliac vein. ${ }^{3}$ Subsequently, May and Thurner described compression of the left common iliac vein from the right common iliac artery, resulting in mural spur formation. ${ }^{4}$

\section{Pathology}

The left common iliac vein has a more transverse or horizontal course compared with the right one and can be compressed by the right common iliac artery against the lumbar spine with each arterial pulsation. This results in increased pressure within the vein and results in symptoms of venous insufficiency, such as leg edema and pain. Chronic repetitive compression leads to secondary venous luminal changes and mural spur formation (secondary to elastin and collagen deposition) with subsequent venous stenosis. A cadaveric study $(n=430)$ demonstrated $22 \%$ of obstructive lesions including lateral spur, central spur, and partial obliterations or multiple fenestrations. ${ }^{4}$ The intimal hyperplasia and stenosis result in a slow but turbulent flow precipitating thrombus formation within the iliac vein. This thrombus in association with the stenosis can result in distal propagation of thrombus, postthrombotic syndrome, and venous insufficiency. Collaterals are formed within the abdomen and pelvis (paralumbar, internal iliac to internal iliac, and so on) to bypass the areas of venous obstruction ( $\mathbf{- F i g . ~} \mathbf{1}$ ).

\section{Clinical Presentation}

Left iliac vein compression is a common finding on imaging; however, most of the patients are asymptomatic. This is often called the May-Thurner anatomy (MTA) in contrast to MTS, which represents symptomatic deep venous thrombosis (DVT) secondary to iliac vein compression. Nazzal et al
DOI https://doi.org/ 10.1055/s-0036-1597954. ISSN 0000-0000.
Copyright $\odot 2017$ by Indian Society of Vascular and Interventional Radiology 


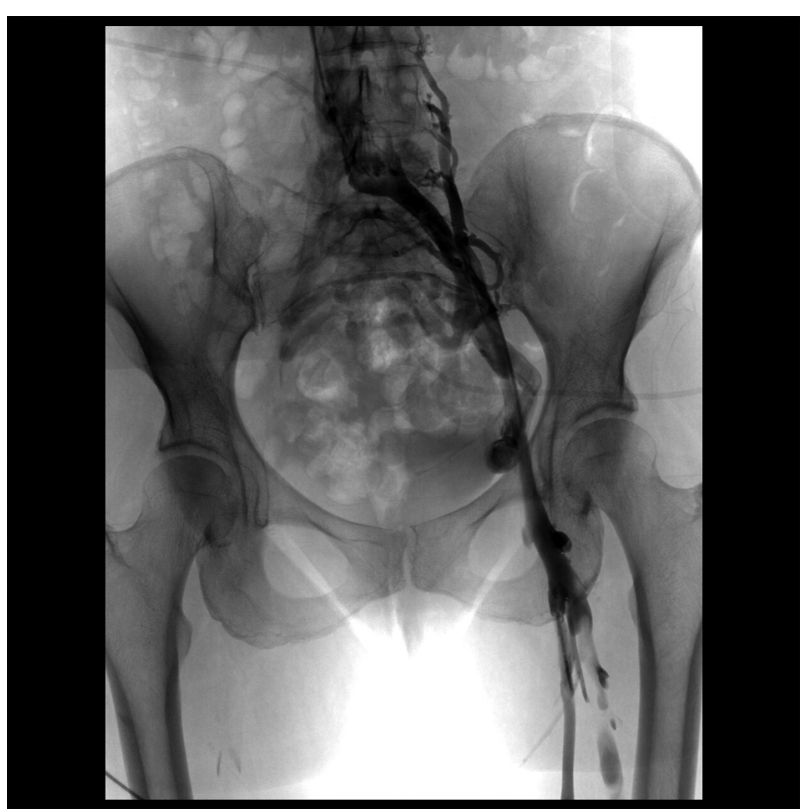

Fig. 1 Contrast venography through left femoral vein demonstrates stenosis at the junction of the left common iliac vein and inferior vena cava. Multiple ascending lumbar and crosspelvic collaterals are identified. These findings are consistent with May-Thurner syndrome in this middle-aged female patient presenting with leg edema and pain without a demonstrable mass lesion on cross-sectional imaging.

reviewed computed tomography (CT) scan images $(n=300)$ and reported asymptomatic iliac vein stenosis of more than $70 \%$ luminal diameter in $30.6 \%$, and the stenosis in itself was not associated with the presence of clinical symptoms. ${ }^{5}$ Kibbe et al reported similar findings: $50 \%$ compression in $24 \%$, and $25 \%$ compression in $66 \%$ of asymptomatic population. ${ }^{6}$ However, another study reported possible association of DVT in patients with greater than $70 \%$ iliac vein compression. ${ }^{7}$ MTS occurs when there is hemodynamically significant venous compression of an underlying MTA from chronic venous changes, and the exact threshold at which it triggers symptomatic DVT is unknown. ${ }^{8}$ This explains the high prevalence of MTA but lower prevalence of clinically evident MTS: 2 to $5 \%$ of all lower extremity venous disorders and 18 to $49 \%$ of left lower limb DVT. ${ }^{9}$ Precipitating factors such as trauma, oral contraceptives, and malignancy can unmask or exacerbate underlying MTS.

Clinical symptoms can vary based on the underlying thrombotic or nonthrombotic presentation. The classic presentation is a young to middle-aged patient (70-90\% female preponderance) presenting with acute left lower extremity DVT without identifiable cause of hypercoagulability. The most frequent symptoms are pain and edema due to decreased venous outflow from the left lower extremity. Chronic MTS might present as venous insufficiency with varicose veins, skin pigmentation, lipodermatosclerosis, and venous ulcers. Similarly, increased pelvic collaterals can result in pelvic varicosities and pelvic venous congestion syndrome. Postthrombotic syndrome might be the presentation in patients with recurrent deep vein thrombosis or incompletely treated MTS. Recurrent DVT occurs in patients in whom the precipitating factor for DVT has been corrected but the underlying MTA has not been addressed. Pulmonary embolism $(\mathrm{PE})$ as the initial presentation is rare, but has been reported. ${ }^{10}$ Patients with spontaneous iliac venous rupture and retroperitoneal hematoma need to be investigated with high suspicion as $28 \%$ have underlying MTS. ${ }^{11}$ Symptoms might also include tingling and numbness in the lumbar area secondary to compression of the neural structures from the enlarged collaterals. A right-sided variant of MTS has been described in patients with dextrocardia, left-sided inferior vena cava (IVC), and compression from the right internal iliac artery. ${ }^{12-14}$ There is an iatrogenic case of MTS reported secondary to placement of an aortic stent graft. ${ }^{15}$ Fretz and Binkert reported a variant MTS with compression of the IVC by right common iliac artery in a patient with high aortic bifurcation. ${ }^{16}$

\section{Diagnosis}

Doppler ultrasound is the initial test of choice to evaluate a patient presenting with left lower extremity edema or pain. Sonographic evaluation is a quick and effective means to diagnose lower extremity DVT with high sensitivity and specificity. Diagnostic findings include increased intraluminal echogenicity, lack of compressibility, and absence of flow on color flow imaging. Direct evaluation of the iliac vein within the pelvis might be difficult due to bowel gas and adjacent overlying structures, but adequate visualization of external iliac and common iliac veins has been reported in 79 and $47 \%$ of the cases, respectively. ${ }^{17}$ Thrombus with absence of flow or hemodynamically significant stenosis (50\% luminal stenosis or peak velocity ratio $>2.5$ compared with the distal segment) provide direct evidence of iliac venous pathology. The absence of respiratory phasicity, mosaic turbulent flow, or lack of response to Valsalva maneuver within the common femoral vein provide an indirect evidence of underlying proximal iliac vein thrombosis (-Fig. 2A). ${ }^{18}$ CT venography provides anatomical detail, excludes other causes of compression, and defines the extent of underlying thrombus and stenosis. Magnetic resonance venography provides information similar to CT venography with better characterization of the pathology as well as additional assessment of hemodynamic significance by demonstrating pelvic collaterals and flow reversal (on time-of-flight pulse sequence) within the ascending lumbar vein. ${ }^{19}$ Contrast venography is the gold standard for diagnosis and provides an opportunity for treatment of MTS in the same setting (-Fig. 2). Venography findings include acute thrombosis within the iliac vein or the iliofemoral veins and stenosis or occlusion of left common iliac vein with pelvic and retroperitoneal collaterals in chronic cases. May and Thurner advocated measurement of pressure differential between the iliac veins $(2 \mathrm{~mm} \mathrm{Hg}$ at rest, $3 \mathrm{~mm}$ $\mathrm{Hg}$ during exercise with exaggerated response on exercise) for diagnosis, whereas Ferris et al advocated any gradient between the IVC and left iliac vein for diagnosis of hemodynamically significant obstruction. ${ }^{4,20}$ Iliocaval venous pressure measurements would also be helpful in the evaluation of in-stent stenosis and response to treatment. The role of intravascular ultrasound (IVUS) is described separately. 


\section{Management}

Systemic anticoagulation is initiated immediately to prevent new or propagation of thrombus. However, it is not effective alone in the management of MTS as the underlying pathology is not addressed. Symptomatic relief can be obtained with the use of compression stockings in chronic cases. Management of MTS is predominantly endovascular aiming at (1) resolving thrombus if acute or subacute, (2) recanalization of occluded or stenotic venous segments, and (3) balloon dilatation and stent placement to maintain the patency of the iliac vein. Endovascular treatment of acute and subacute thrombus involves catheter directed pharmacological thrombolysis and mechanical thrombectomy, either alone or in combination. Subsequent stenting of the iliac vein is required. Chronic
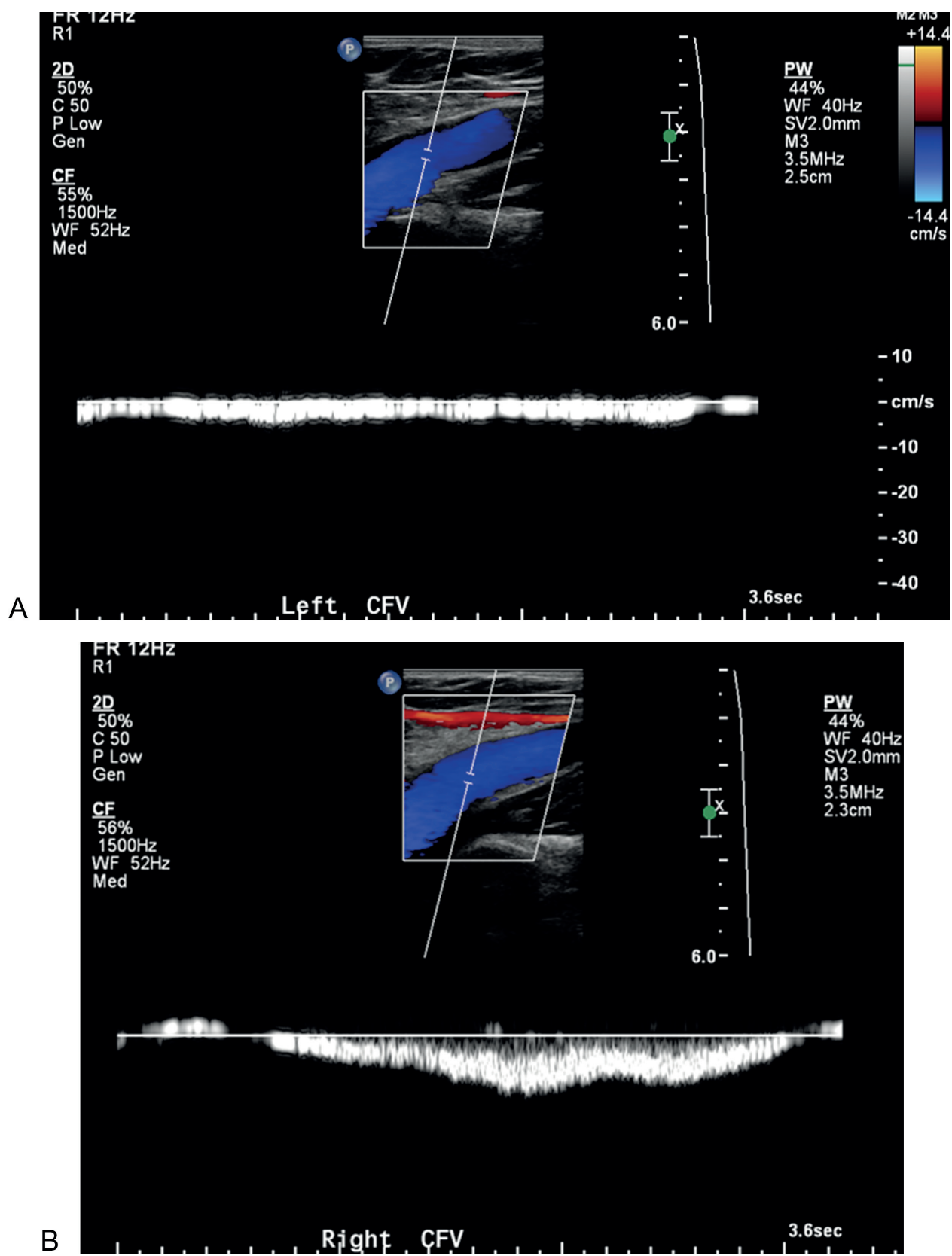

Fig. 2 A young female with a history of recurrent episodes of deep venous thrombosis on medical treatment alone now presenting with left lower leg symptoms. Doppler ultrasound (US) evaluation of left common femoral vein (A) demonstrates lack of respiratory phasicity compared with right common femoral vein (B), indicating a proximal left iliac venous stenosis or occlusion. (C) Venography demonstrates stenosis of left common iliac vein at its confluence with the inferior vena cava (IVC) suggestive of May-Thurner syndrome. Multiple collaterals are also identified indicating the chronic nature. Left common iliac pressure was $6 \mathrm{~mm} \mathrm{Hg}$ (right iliac vein and IVC pressures are $4 \mathrm{~mm} \mathrm{Hg}$ ) confirming venography findings of a hemodynamic stenosis. (D) Balloon dilatation was performed followed by deployment of a 16-mm noncovered self-expanding stainless steel stent resulting in restored caliber and flow. Pressure measurements normalized subsequent to intervention. (E) Contrast-enhanced axial venous phase computed tomography scan image demonstrates a patent stent. (F) Postprocedural follow-up US illustrates restored respiratory phasicity within the left common femoral vein indicating patency of the left common iliac stent. 

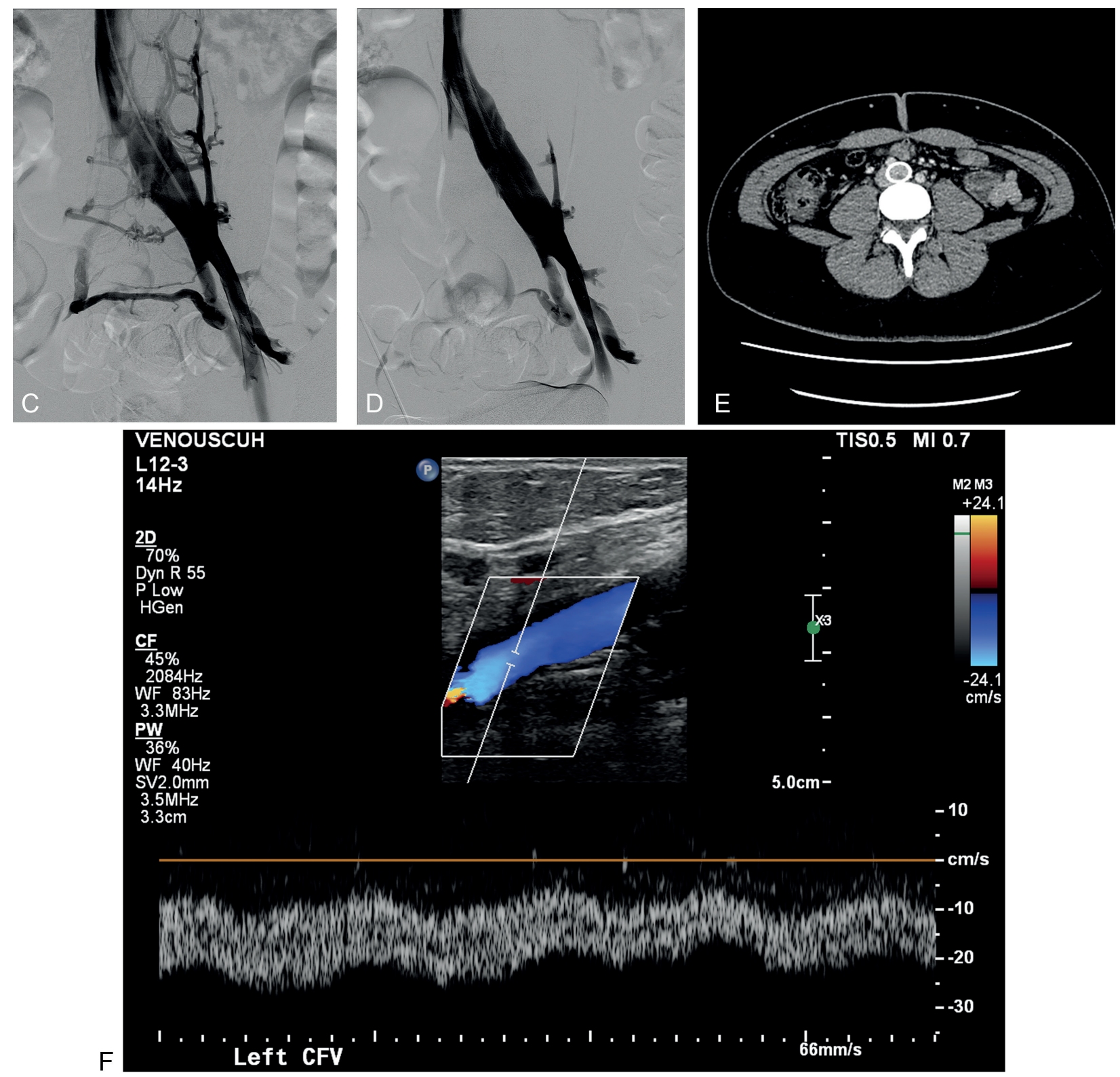

Fig. 2 (Continued)

thrombotic and stenotic lesions usually require percutaneous balloon dilation and stenting. Surgical outcomes are not favorable compared with endovascular intervention due to higher mortality and morbidity, and shorter patency rates of venous bypass grafts. Surgical management is only indicated in patients with failed endovascular treatment and involves vein repair with thrombus removal, relocation of the artery, and placement of a venous bypass graft. ${ }^{21,22}$

\section{Endovascular Interventions}

The patient is usually positioned prone and an antegrade ipsilateral popliteal venous access is obtained under US guidance. Popliteal venous access is preferred (compared with contralateral femoral or right jugular access) as antegrade access is atraumatic to venous valves with the use of wires and catheters. Additionally, it provides an opportunity to treat the peripheral extension of the iliac venous thrombus from the same access site. An extensive thrombotic involvement of the popliteal vein might necessitate posterior tibial or small saphenous vein access. Isolated iliac vein involvement can be treated with femoral vein access. Therefore, a preprocedural US study to evaluate the extent of thrombus is an essential step in planning the access site. If the thrombus is soft and recanalization across the iliac vein is performed without any difficulty, a pullback venography demonstrates the central extent of clot as filling defects and serves to exclude IVC thrombus. An underlying stenosis may not be evident in the presence of acute thrombus. Occasionally, crossing the stenosis might not be possible necessitating initiation of 
thrombolysis or thrombectomy in the iliofemoral and distal venous segments.

Catheter-directed thrombolysis is initiated with injection of thrombolytics such as Alteplase (tPA, Activase, Cathflo, Activase; Genentech Inc., San Francisco, California) through a multiside hole infusion catheter. Mechanical thrombectomy with balloon maceration, and rheolytic thrombectomy with the AngioJet device (Possis Medical, Minneapolis, Minnesota), Trellis device (Covidien, Dublin, Ireland), or EKOS Endowave system (EKOS Corporation, Bothell, Washington) are very effective in addressing acute and subacute thrombus and are preferred over pharmacological thrombolysis alone. If the thrombus resolves promptly, treatment of the underlying iliac vein stenosis is performed with balloon dilatation and stent placement. Residual thrombus requires continued pharmacological thrombolysis with an appropriate infusion catheter (Unifuse, AngioDynamics, Queensbury, New York; CraggMcNamara; Micro Therapeutics, Irvine, California) across the thrombus. Alteplase is administered at 0.5 to $1 \mathrm{mg} /$ hour through the infusion catheter and heparin is administered at 500 to 1,000 units/hour through the sheath or through a peripheral intravenous (IV) cannula. Thrombolysis is continued in an intensive care unit setting for 24 to 48 hours with continuous monitoring of fibrinogen levels and complete blood count. A repeat venography is performed to assess interval resolution of thrombus and to define the extent of venous stenosis. Residual thrombus would necessitate continuation of thrombolytic therapy, unless contraindicated; however, a prolonged but failed infusion therapy suggests refractory chronic thrombus and treatment failure. It is important to obtain complete resolution of the thrombus for optimal outcomes. Recanalization is performed at this time, if failed initially. After resolution of the thrombus, the underlying obstruction is evaluated and characterized. Balloon dilation is performed followed by deployment of selfexpanding stent within the stenosed iliac vein. Treatment of chronic iliac venous stenosis or isolated chronic refractory common iliac thrombus involves balloon dilatation and stent placement (-Fig. $\mathbf{3}$ ).

\section{Stent Placement}

Placement of a stent across venous stenosis has been proven to be beneficial compared with balloon dilation alone and is in fact the corner stone for successful management of symptomatic MTS. ${ }^{23}$ Currently, there is no evidence supporting prophylactic stenting in asymptomatic patients with MTA. A self-expanding 12 to $16 \mathrm{~mm}$ noncovered stent (Wallstent, Boston Scientific, Marlborough, Massachusetts; Smart Stent, Cordis, Milpitas, California; Zilver Vena, Cook Medical, Bloomington, Indiana) is preferred and deployed across the stenosis. Stainless steel stents provide higher radial force, but choice of a particular stent is highly dependent on institutional and individual preference. Self-expanding stents are preferred compared with balloon mounted stents given their flexibility, longer length, durability. and ability to not deform with arterial pulsations. In cases where dilatation of the lesion cannot be achieved in preparation for stent deployment or if the self- expanding stent fails to achieve necessary patency, balloon mounted stents can be useful. Slight proximal extension of the stent into the IVC is preferred to serve as an anchor and prevents stent migration. ${ }^{24,25}$ Extension of the stent into the external iliac (-Fig. 4) and femoral veins might be necessary in patients with thrombus extension, though extension peripheral or caudal to the inguinal ligament has been associated with higher rates of in-stent stenosis and thrombosis. ${ }^{24,26}$

\section{Role of Intravascular Ultrasound}

Intravascular ultrasound improves diagnostic accuracy and is helpful in characterizing the intimal lesions and partial or mural adherent thrombus (-Fig. 3D). Dynamic compression of the iliac vein by the iliac artery can be demonstrated. Underlying spurs, webs, channels, and stenosis are identified in chronic cases. ${ }^{27,28}$ Recanalization in difficult cases can be performed with IVUS guidance. Accurate evaluation of the extent of venous stenosis and residual thrombus is very helpful for planning endovascular treatment. Additionally, there might be a role for evaluating response to balloon dilatation and stent placement. ${ }^{29}$

\section{Use of Inferior Vena Cava Filter}

There is no supporting evidence to recommend routine use of an IVC filter prior to iliac venous interventions. ${ }^{23}$ Placement of an IVC filter prior to thrombolytic intervention has been anecdotally proposed for individuals with limited cardiopulmonary reserve, true free floating thrombus, recurrent PE in spite of anticoagulation, and high thrombus burden. ${ }^{30}$ Moreover, the incidence of PE is lower in MTS, probably secondary to the intraluminal synechia acting as a filter to prevent migration of thrombus into the lungs. Chan et al reported an $83 \%$ lower risk of developing symptomatic PE in patients with unilateral DVT and ipsilateral common iliac vein measuring $4 \mathrm{~mm}$ or less. ${ }^{31}$

\section{Postprocedure Management and Follow-Up}

Procedure-related complications include access site complications and intra-abdominal or retroperitoneal bleeding. Complications related to the use of thrombolytic drugs include bleeding such as intracranial or gastrointestinal hemorrhage and disseminated intravascular coagulation. PE is a possible complication at the time of endovascular interventions. Long-term complications of endovascular stenting include in-stent stenosis, migration, and fracture-related complications (-Fig. 5). Systemic anticoagulation (loading dose of clopidogrel followed by maintenance dose for 6-12 weeks, heparin IV with transition to warfarin or low-molecular-weight heparin for 3-6 months, and acetylsalicylic acid indefinitely) is recommended following stent placement. Long-term gradient compression stockings (30-40 $\mathrm{mm} \mathrm{Hg}$ ) are also suggested to prevent postthrombotic syndrome. Clinical and imaging follow-up is performed at frequent intervals to ensure that the patient is symptom-free. Any recurrence of symptoms should prompt imaging or venography. Shi et al reported primary patency rates of $93.2,84.3$, and $74.5 \%$ and 

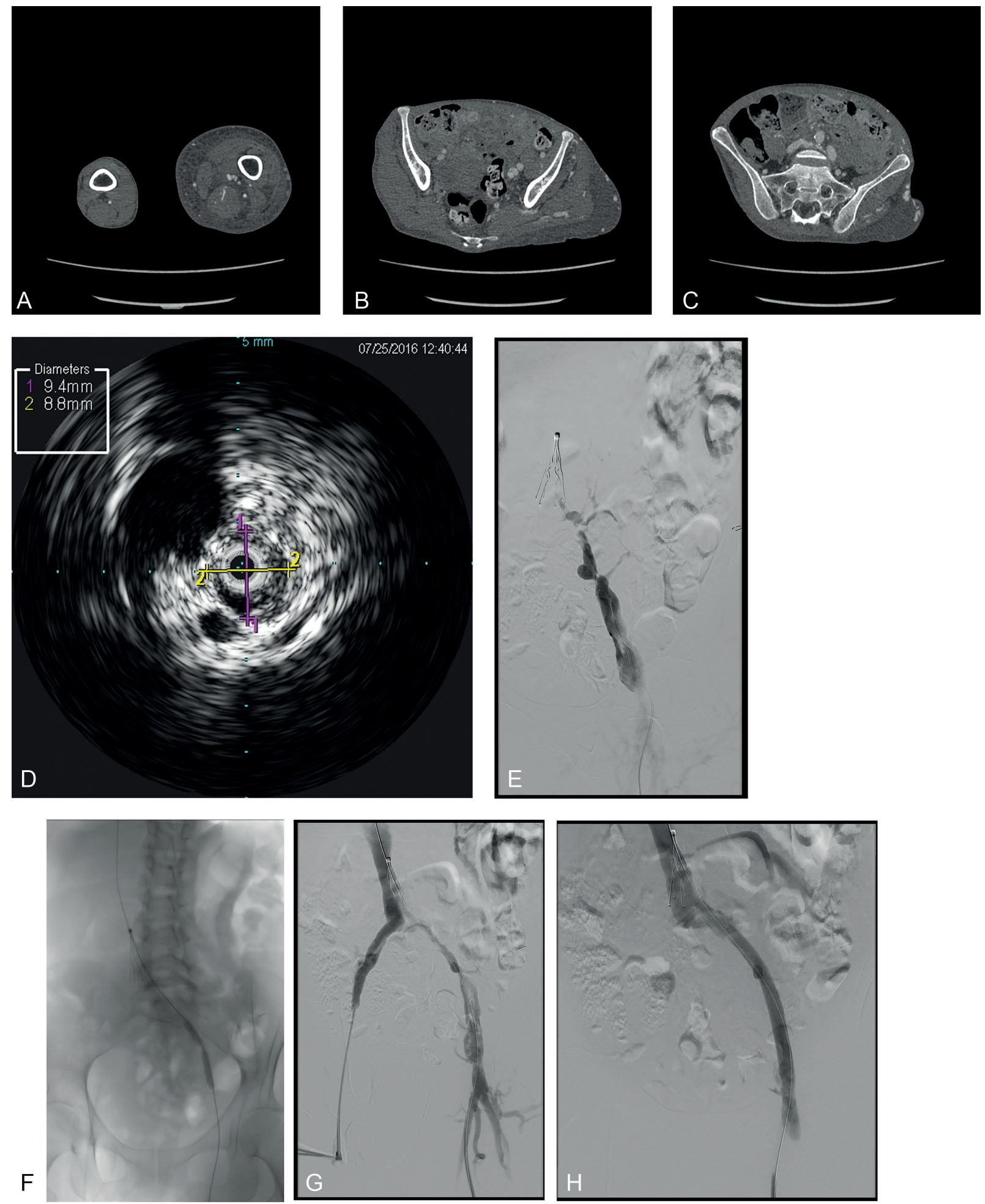

Fig. 3 A middle-aged woman presenting with chronic severe leg pain and swelling. Computed tomography scan demonstrates significant symmetrically enlarged left lower extremity (A) as well as multiple venous collaterals (B), and a May-Thurner anatomy (C) with compression of the left common iliac vein by the right common iliac artery. (D) Intravascular ultrasound demonstrates narrowed venous lumen and adjacent compression from the artery. Contrast venography demonstrates severe stenosis $(\mathrm{E})$, and the stenosis was treated with balloon dilatation $(\mathrm{F})$ with persistent stenosis and small residual thrombus $(G)$. Subsequently, a 14-mm stent was deployed with restored flow and no residual stenosis (H). Endovascular intervention with placement of a stent after resolution of thrombus, if any, is the first-line choice for the treatment of May-Thurner syndrome. 

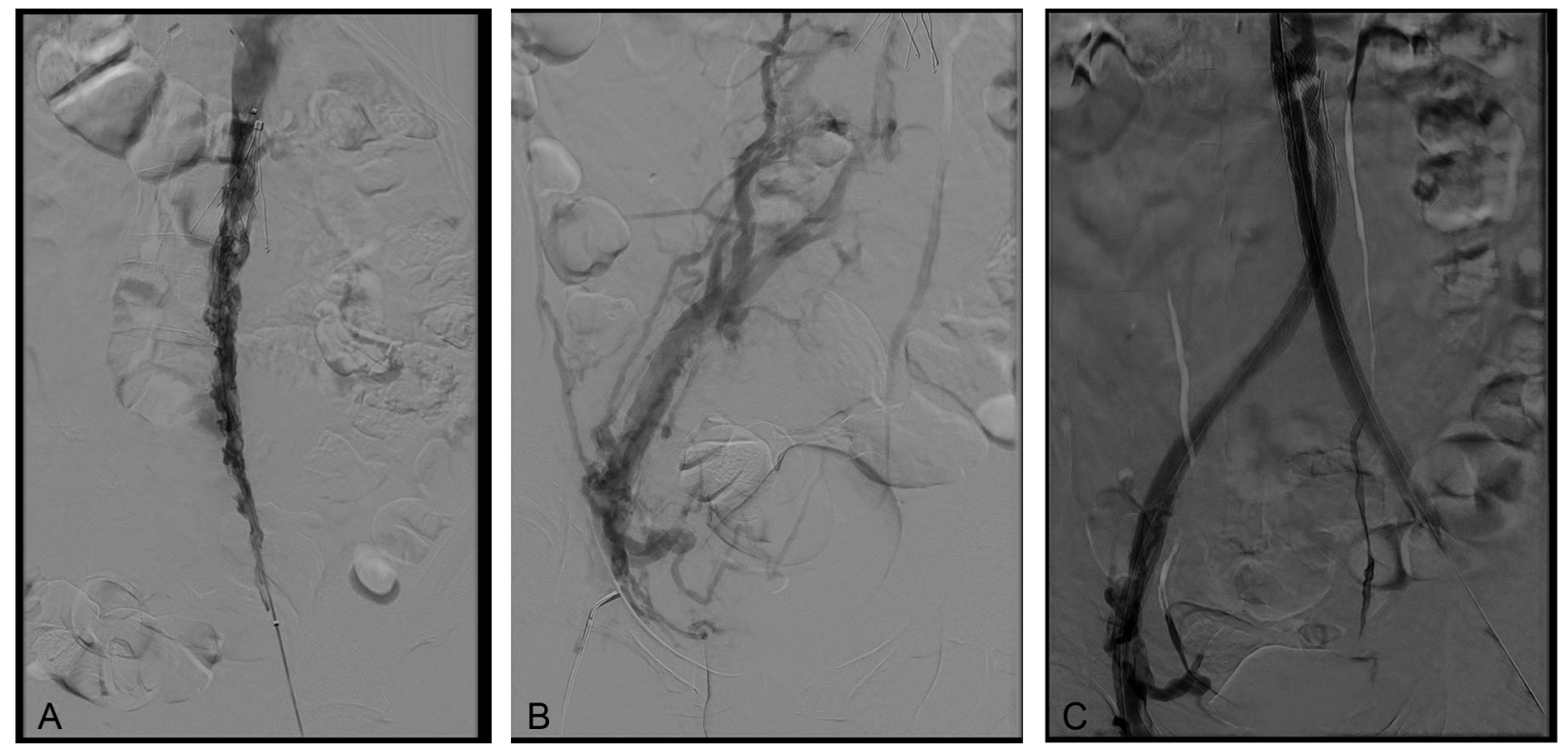

Fig. 4 (A) Contrast venography demonstrates filling defects within the inferior vena cava (IVC) consistent with thrombus and stenosis. (B) MayThurner anatomy is identified on the left with stenosis of left common iliac vein. Filling defects were also noted to extend into the central right common iliac vein. A wire was advanced through each femoral vein, extending through the same gap within the IVC filter legs. Two self-expanding stents were simultaneously deployed within the IVC and bilateral common iliac veins and subsequently extended to external iliac veins. (C) Completion venogram demonstrates restored flow and caliber in the iliac veins and IVC.
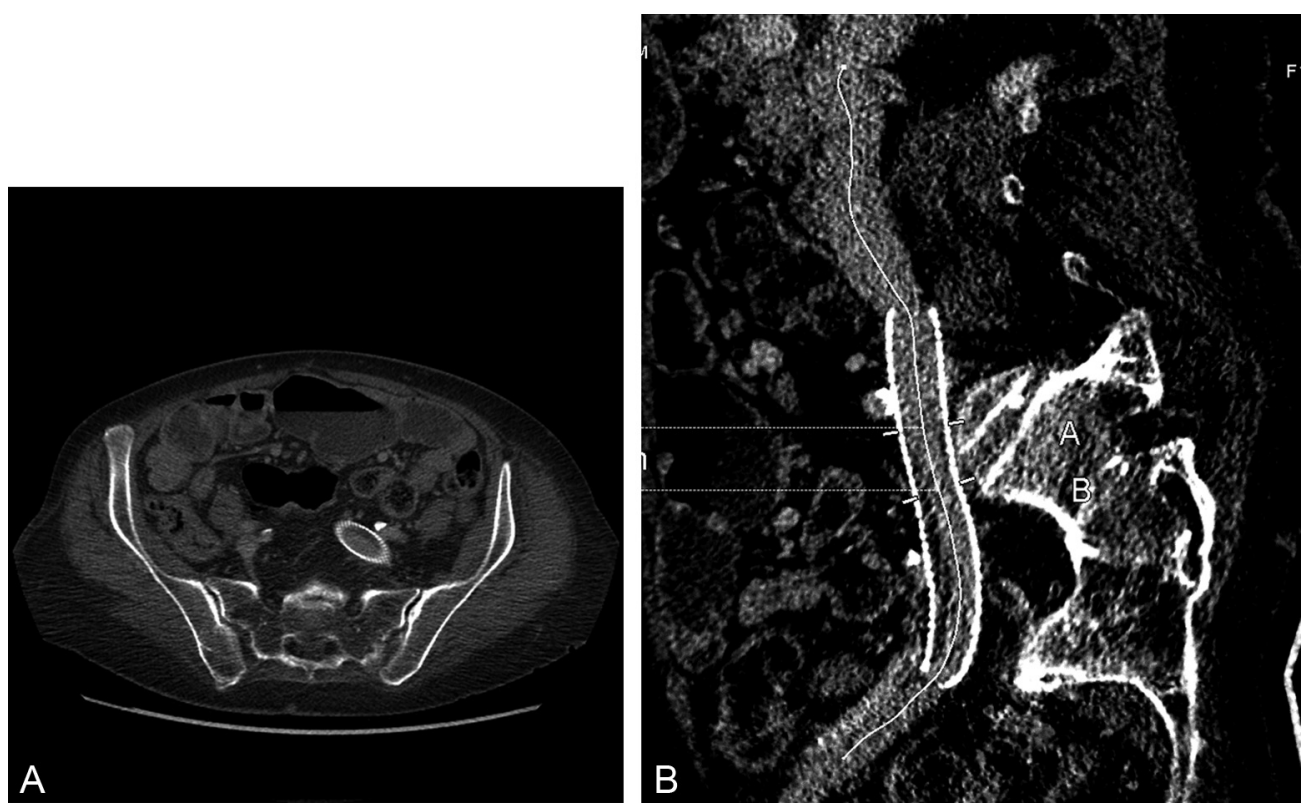

Fig. 5 Recurrence of symptoms in patient with previous endovascular treatment of May-Thurner syndrome should prompt investigation for occlusion or thrombus. (A,B) Images demonstrate a less than $50 \%$ stenosis with chronic thrombotic changes in a patient with previously stented May-Thurner syndrome on surveillance imaging. Based on the clinical and imaging presentation, thrombectomy and balloon dilation with or without stenting might be necessary to maintain patency. Pressure measurement might be helpful in equivocal cases.

secondary patency rates of $100,93.3$, and $92 \%$ at 1,3 , and 5 years, respectively, for iliac vein stents in compression syndrome. ${ }^{32}$ This study also reported use of multiple stents and irregular stock wearing as independent predictors for in-stent stenosis. A recent meta-analysis (37 studies, 2,869 patients) reported a technical success of 94 to $96 \%$ and 1 year primary and secondary stent patency rates (nonthrombotic: 96 and 99\%; acute thrombotic: 87 and 89\%; chronic postthrombotic: 79 and $94 \%$, respectively) for iliofemoral obstruction. $^{33}$ Thrombus or in-stent stenosis would require pharmacomechanical thrombectomy and balloon dilatation with or without additional stent placement. 


\section{Conclusion}

May-Thurner syndrome is a common cause of left lower extremity deep vein thrombosis; however, it is underdiagnosed. In patients presenting with left-sided DVT without obvious etiology, especially in young women, a high clinical suspicion is needed for prompt diagnosis of MTS. Endovascular intervention with thrombolysis and stenting remains the first choice for successful outcomes. Early recognition and aggressive treatment are vital for symptomatic relief and to prevent recurrent thrombosis and postthrombotic syndrome.

\section{References}

1 Cockett FB, Thomas ML. The iliac compression syndrome. Br J Surg 1965;52(10):816-821

2 McMurrich JP. The occurrence of congenital adhesions in the common liac veins, and their relation to thrombosis of the femoral and iliac veins. Am J Med Sci 1908;135(3):342-345

3 Ehrich WE. A frequent obstructive anomaly of the mouth of the left common iliac vein. Am Heart J 1943;26:18-31

4 May R, Thurner J. The cause of the predominantly sinistral occurrence of thrombosis of the pelvic veins. Angiology 1957;8(5): 419-427

5 Nazzal M, El-Fedaly M, Kazan V, et al. Incidence and clinical significance of iliac vein compression. Vascular 2015;23(4): 337-343

6 Kibbe MR, Ujiki M, Goodwin AL, Eskandari M, Yao J, Matsumura J. Iliac vein compression in an asymptomatic patient population. J Vasc Surg 2004;39(5):937-943

7 Narayan A, Eng J, Carmi L, et al. Iliac vein compression as risk factor for left- versus right-sided deep venous thrombosis: case-control study. Radiology 2012;265(3):949-957

8 Birn J, Vedantham S. May-Thurner syndrome and other obstructive iliac vein lesions: meaning, myth, and mystery. Vasc Med 2015;20(1):74-83

9 Bozkaya H, Cinar C, Ertugay S, et al. Endovascular treatment of iliac vein compression (May-Thurner) syndrome: angioplasty and stenting with or without manual aspiration thrombectomy and catheter-directed thrombolysis. Ann Vasc Dis 2015;8(1):21-28

10 Fasanya AA, LaCapra G. May-Thurner syndrome with pulmonary embolism as the first presentation rather than deep vein thrombosis. Cureus 2016;8(2):e509

11 Jiang J, Ding X, Zhang G, Su Q Wang Z, Hu S. Spontaneous retroperitoneal hematoma associated with iliac vein rupture. J Vasc Surg 2010;52(5):1278-1282

12 Vijayalakshmi IB, Setty HS, Narasimhan C. Unusual cases of rightsided and left-sided May-Thurner syndrome. Cardiol Young 2015; 25(4):797-799

13 Burke RM, Rayan SS, Kasirajan K, Chaikof EL, Milner R. Unusual case of right-sided May-Thurner syndrome and review of its management. Vascular 2006;14(1):47-50

14 Molloy S, Jacob S, Buckenham T, Khaw KT, Taylor RS. Arterial compression of the right common iliac vein; an unusual anatomical variant. Cardiovasc Surg 2002;10(3):291-292

15 Pandit AS, Hayes M, Guiney-Borgelt S, Dietzek AM. Iatrogenic MayThurner syndrome after EVAR. Ann Vasc Surg 2014;28(3):739. e17-739.e20
16 Fretz V, Binkert CA. Compression of the inferior vena cava by the right iliac artery: a rare variant of May-Thurner syndrome. Cardiovasc Intervent Radiol 2010;33(5):1060-1063

17 Messina LM, Sarpa MS, Smith MA, Greenfield LJ. Clinical significance of routine imaging of iliac and calf veins by color flow duplex scanning in patients suspected of having acute lower extremity deep venous thrombosis. Surgery 1993;114(5):921-927

18 Labropoulos N, Borge M, Pierce K, Pappas PJ. Criteria for defining significant central vein stenosis with duplex ultrasound. J Vasc Surg 2007;46(1):101-107

19 Wolpert LM, Rahmani O, Stein B, Gallagher JJ, Drezner AD. Magnetic resonance venography in the diagnosis and management of MayThurner syndrome. Vasc Endovascular Surg 2002;36(1):51-57

20 Ferris EJ, Lim WN, Smith PL, Casali R. May-Thurner syndrome. Radiology 1983;147(1):29-31

21 Morelli MM, Burchi C, Battistini M, Capone AM, Cavallaro A. Venovenous crossover bypass according to Palma: our experience [in Italian]. Ann Ital Chir 1996;67(4):521-525, discussion 525-526

22 Jost CJ, Gloviczki P, Cherry KJ Jr, et al. Surgical reconstruction of iliofemoral veins and the inferior vena cava for nonmalignant occlusive disease. J Vasc Surg 2001;33(2):320-327, discussion 327-328

23 Meissner MH, Gloviczki P, Comerota AJ, et al; Society for Vascular Surgery; American Venous Forum. Early thrombus removal strategies for acute deep venous thrombosis: clinical practice guidelines of the Society for Vascular Surgery and the American Venous Forum. J Vasc Surg 2012;55(5):1449-1462

24 O'Sullivan GJ, Semba CP, Bittner CA, et al. Endovascular management of iliac vein compression (May-Thurner) syndrome. J Vasc Interv Radiol 2000;11(7):823-836

25 Suwanabol PA, Tefera G, Schwarze ML. Syndromes associated with the deep veins: phlegmasia cerulea dolens, May-Thurner syndrome, and nutcracker syndrome. Perspect Vasc Surg Endovasc Ther 2010;22(4):223-230

26 Sapoval MR, Long AL, Raynaud AC, Beyssen BM, Fiessinger JN, Gaux JC. Femoropopliteal stent placement: long-term results. Radiology 1992;184(3):833-839

27 Ahmed HK, Hagspiel KD. Intravascular ultrasonographic findings in May-Thurner syndrome (iliac vein compression syndrome). J Ultrasound Med 2001;20(3):251-256

28 Butros SR, Liu R, Oliveira GR, Ganguli S, Kalva S. Venous compression syndromes: clinical features, imaging findings and management. Br J Radiol 2013;86(1030):20130284

29 Forauer AR, Gemmete JJ, Dasika NL, Cho KJ, Williams DM. Intravascular ultrasound in the diagnosis and treatment of iliac vein compression (May-Thurner) syndrome. J Vasc Interv Radiol 2002; 13(5):523-527

30 Moudgill N, Hager E, Gonsalves C, Larson R, Lombardi J, DiMuzio P. May-Thurner syndrome: case report and review of the literature involving modern endovascular therapy. Vascular 2009;17(6): 330-335

31 Chan KT, Popat RA, Sze DY, et al. Common iliac vein stenosis and risk of symptomatic pulmonary embolism: an inverse correlation. J Vasc Interv Radiol 2011;22(2):133-141

32 Shi WY, Gu JP, Liu CJ, He X, Lou WS. Endovascular treatment for iliac vein compression syndrome with or without lower extremity deep vein thrombosis: a retrospective study on mid-term in-stent patency from a single center. Eur J Radiol 2016;85(1):7-14

33 Razavi MK, Jaff MR, Miller LE. Safety and effectiveness of stent placement for iliofemoral venous outflow obstruction: systematic review and meta-analysis. Circ Cardiovasc Interv 2015;8(10): e002772 